\title{
Burrowing activity of the Neohelice granulata crab (Brachyura, Varunidae) in southwest Atlantic intertidal areas
}

\section{Actividad cavadora del cangrejo Neohelice granulata (Brachyura, Varunidae) en sitios intermareales del atlántico sudoccidental}

\author{
Sabrina Angeletti ${ }^{1 *}$, Patricia M Cervellini ${ }^{1}$, Leticia Lescano ${ }^{2,3}$ \\ ${ }^{1}$ Instituto de Ciencias Biológicas y Biomédicas del Sur, Consejo Nacional de Investigaciones Científicas y \\ Técnicas-Universidad Nacional del Sur (CONICET-UNS), San Juan 670, 8000-Bahía Blanca, Argentina. \\ 2 Departamento de Geología, Universidad Nacional del Sur, San Juan 670, 8000-Bahía Blanca, Argentina. \\ ${ }^{3}$ Comisión de Investigaciones Científicas de la Provincia de Buenos Aires, Calle 526 entre 10 y 11, 1900-La \\ Plata, Argentina. \\ * Corresponding author. E-mail: angelettisabrina@conicet.gov.ar
}

\begin{abstract}
The burrowing and semiterrestrial crab Neohelice granulata actively and constantly builds its burrows in the intertidal zone of the Bahía Blanca Estuary during low tide. Differences in structural morphology of $N$. granulata burrows and burrowing activities in contrasting microhabitats (saltmarsh and mudflat) were analyzed and related to several conditions, such as tide level, substrate type, sediment properties, and population density. In the mudflat the higher density of total burrows in autumn ( 172 burrows $\left.\cdot \mathrm{m}^{-2}\right)$ was associated with molt timing, and the higher density of active burrows in summer $\left(144\right.$ burrows $\left.\cdot \mathrm{m}^{-2}\right)$ was associated with reproductive migration. Sediments from biogenic mounds (removed by crabs) showed higher water content and penetrability than surface sediments (control), suggesting that bioturbation increases the values of these parameters. Grain size distribution profiles and mineralogical composition did not vary between microhabitats or between seasons. Burrows were all tunnel-shaped and only those from the saltmarsh had chambers inside the tunnels, possibly used for reproductive purposes. Burrows from the saltmarsh had narrow entrances and deep tunnels, and burrows from the mudflat presented very narrow entrances and surface tunnels. Differences in burrow features and burrow activity of $N$. granulata between microhabitats were confirmed and associated with biotic and abiotic factors, indicating that this species has an adaptive burrowing behavior.
\end{abstract}

Keywords: bioturbation, burrowing organisms, brackish-water environment, ecological zonation, sediments.

RESUMEN. El cangrejo cavador y semiterrestre Neohelice granulata construye sus cuevas activamente y constantemente en la zona intermareal del estuario de Bahía Blanca durante la marea baja. Se analizaron diferencias en la morfología estructural de las cuevas y la actividad excavadora de N. granulata en microhábitats contrastantes (marisma y planicie de marea) y se relacionaron con diversas condiciones, como el nivel de las mareas, el tipo de sustrato, las propiedades del sedimento y la densidad poblacional. En la planicie de marea la mayor densidad de cuevas totales en el otoño (172 cuevas $\cdot \mathrm{m}^{-2}$ ) se relacionó con el fenómeno de muda, y la mayor densidad de cuevas activas durante el verano (144 cuevas $\cdot \mathrm{m}^{-2}$ ) se relacionó con la migración reproductiva. Los sedimentos de los montículos biogénicos (removidos por cangrejos) presentaron mayor contenido de agua y penetrabilidad que los sedimentos superficiales (control), lo cual sugiere que la bioturbación incrementa los valores de estos parámetros. La granulometría y mineralogía no variaron entre los microhábitats ni entre las estaciones. Todas las cuevas presentaron forma de túnel y sólo aquellas provenientes de la marisma presentaron cámaras dentro de los túneles, posiblemente usadas con propósitos reproductivos. Las cuevas de la marisma exhibieron entradas angostas y túneles profundos, y las de la planicie de marea presentaron entradas muy angostas y túneles superficiales. Se confirmaron diferencias en las características de las cuevas y en la actividad excavadora de $N$. granulata entre los distintos ambientes y se relacionaron con los factores bióticos y abióticos, lo cual indica que esta especie tiene un comportamiento excavador adaptativo.

Palabras clave: bioturbación, organismos excavadores, ambientes salobres, zonación ecológica, sedimentos.

\section{INTRODUCTION}

Neohelice granulata is a crab with a characteristic burrowing behavior. It digs and keeps open semipermanent burrows, allowing it to adopt a semiterrestrial life from the northerneastern coast of Patagonia, Argentina $\left(42^{\circ} 25^{\prime} \mathrm{S}\right.$, $\left.64^{\circ} 36^{\prime} \mathrm{W}\right)$, to Río de Janeiro, Brazil $\left(22^{\circ} 57^{\prime} \mathrm{S}, 42^{\circ} 50^{\prime} \mathrm{W}\right)$ (Spivak 2010). This species uses these burrows to protect itself

\section{INTRODUCCIÓN}

Neohelice granulata es un cangrejo con un comportamiento excavador característico. Excava y mantiene abiertas sus cuevas semipermanentes, lo que le permite adoptar una existencia semi-terrestre desde la costa noroeste de la Patagonia, Argentina $\left(42^{\circ} 25^{\prime} \mathrm{S}, 64^{\circ} 36^{\prime} \mathrm{W}\right)$, hasta Río de Janeiro, Brasil $\left(22^{\circ} 57^{\prime} \mathrm{S}, 42^{\circ} 50^{\prime} \mathrm{W}\right)$ (Spivak 2010). Esta 
from wave action, extreme temperatures, and desiccation. The burrows also provide shelter from aerial and terrestrial predators during periods of low tide and from aquatic predators at high tide, thus avoiding the stress of finding refuge or escaping predators. Moreover, burrows are the places where 2 important events in a crab's life occur, molting and reproduction, and also where young recruits are safeguarded until they reach larger sizes (Milner et al. 2010, Sal-Moyano et al. 2012).

When building and maintaining their burrows, crabs bring sediments to the surface, forming mounds near burrow entrances (Murray et al. 2002). This bioturbation affects sediment structure, because the cohesive nature of the organic matrix is disrupted by this process. Apart from directly affecting sediment porosity and permeability, this process has ecological significance in the aeration of soils containing anoxic sediments and in the distribution of halophytes. Consequently, high densities of active burrowers can increase erosion rates and sediment mobility (Botto and Iribarne 2000).

Variations in the architecture of $N$. granulata burrows have been observed in studies conducted at other locations in Argentina, such as Mar Chiquita Lagoon, Buenos Aires Province $\left(36^{\circ} 09^{\prime} 26^{\prime \prime} \mathrm{S}, 60^{\circ} 34^{\prime} 11^{\prime \prime} \mathrm{W}\right.$; Iribarne et al. 1997, Botto et al. 2006), and San Antonio Bay, Río Negro Province $\left(40^{\circ} 44^{\prime} 51.43^{\prime \prime} \mathrm{S}\right.$, 64 $52^{\prime} 5.10^{\prime \prime} \mathrm{W}$; Sal-Moyano et al. 2012, Luppi et al. 2013, Bas et al. 2014). These studies revealed differences in terms of size and shape of burrows (diameter, depth, volume, and angle) when different intertidal sites, relative to the tide line, were compared.

In the Bahía Blanca Estuary $\left(30^{\circ} 45^{\prime}-39^{\circ} 25^{\prime} \mathrm{S}, 61^{\circ} 45^{\prime}-\right.$ $62^{\circ} 25^{\prime} \mathrm{W}$ ), which is near the southern range of $N$. granulata, Escapa et al. $(2007,2008)$ analyzed the potential role of the burrowing crab in the erosion of salt marshes and described some architectural variables; their studies are the only antecedents near our study area. However the Bahía Blanca Estuary is a broad ecosystem, and local and specific studies are necessary to include the different habitats in this singular environment. In this context, the main goal of the present study was to assess differences in burrowing behavior and the structural morphology of $N$. granulata burrows at different intertidal sites in a mesotidal estuary. We hypothesized that burrowing activity and burrow morphology would vary between contrasting habitats, which were selected on the basis of their differences in tide level, sediment characteristics, and substrate type.

\section{MATERIALS AND METHODS}

\section{Study area}

The Bahía Blanca Estuary, in the southwest region of the Buenos Aires Province, covers an area of approximately $3,000 \mathrm{~km}^{2}$. It is a mesotidal coastal plain estuary with a semidiurnal tidal regime, and it is characterized by the especie usa las cuevas para protegerse de la acción de las olas, las temperaturas extremas y la desecación. Las cuevas también brindan refugio de los depredadores aéreos y terrestres durante los periodos de marea baja y de los depredadores acuáticos en marea alta, evitando así el estrés de buscar refugio o escapar de los depredadores. Además, estas cuevas son los lugares donde ocurren 2 eventos importantes en la vida del cangrejo, la muda y la reproducción, y también donde los jóvenes reclutas son salvaguardados hasta que alcanzan tamaños más grandes (Milner et al. 2010, Sal Moyano et al. 2012).

Mientras construyen y mantienen sus cuevas, los cangrejos traen sedimentos a la superficie y forman montículos cerca de las entradas (Murray et al. 2002). Esta bioturbación afecta la estructura de los sedimentos, debido a que la naturaleza cohesiva de la matriz orgánica se interrumpe durante este proceso. Además de afectar directamente la porosidad y la permeabilidad de los sedimentos, este proceso tiene gran importancia ecológica al ayudar a airear los suelos que contienen sedimentos anóxicos y a distribuir la flora halófila. En consecuencia, las altas densidades de excavadores activos pueden aumentar las tasas de erosión y la movilidad de los sedimentos (Botto e Iribarne 2000).

En estudios previos en otros lugares de Argentina, como la laguna Mar Chiquita, provincia de Buenos Aires $\left(36^{\circ} 09^{\prime} 26^{\prime \prime} \mathrm{S}, 60^{\circ} 34^{\prime} 11^{\prime \prime} \mathrm{W}\right.$; Iribarne et al. 1997, Botto et al. 2006), y la bahía de San Antonio, provincia de Río Negro (4044'51.43"S, 6452'5.10"W; Sal Moyano et al. 2012, Luppi et al. 2013, Bas et al. 2014), se han observado variaciones en la arquitectura de las cuevas de $N$. granulata. Estos estudios revelaron diferencias en los tamaños y las formas (diámetro, profundidad, volumen y ángulo) de las cuevas cuando diferentes sitios intermareales, con relación a la línea de marea, fueron comparados.

En el estuario de Bahía Blanca $\left(30^{\circ} 45^{\prime}-39^{\circ} 25^{\prime} \mathrm{S}\right.$, $\left.61^{\circ} 45^{\prime}-62^{\circ} 25^{\prime} \mathrm{W}\right)$, cercano al límite sur de la distribución de $N$. granulata, Escapa et al. $(2007,2008)$ analizaron el papel potencial del cangrejo cavador en la erosión de las marismas y describieron algunas variables de la arquitectura de sus cuevas; estos estudios son los únicos antecedentes cerca de nuestra área de estudio. Sin embargo, el estuario de Bahía Blanca es un ecosistema amplio, y se necesitan estudios locales y específicos para incluir los diferentes hábitats dentro de este ambiente singular. En este contexto, el objetivo principal del presente estudio fue evaluar las diferencias en el comportamiento excavador y en la morfología estructural de las cuevas de $N$. granulata en los diferentes sitios intermareales de un estuario mesomareal. Nuestra hipótesis fue que la actividad de excavación y la morfología de las cuevas variarían entre hábitats contrastantes, los cuales fueron seleccionados en función de su diferencia en el nivel de la marea, las características del sedimento y el tipo de sustrato. 
presence of islands that are interconnected by an extensive system of tidal channels that is affected by up to 4-m tides. Freshwater input to the estuary is weak and comes mainly from 2 tributaries on the northern shore of the interior part of the system (Melo 2004). Salinity in the middle portion of the estuary is about 33.98 (annual mean), whereas the inner zone becomes hypersaline during dry summers because of the high evaporation rate (Piccolo et al. 2008). The sampling area was located in Cuatreros Port ( $\left.38^{\circ} 44^{\prime} 50^{\prime \prime} \mathrm{S}, 62^{\circ} 23^{\prime} 5^{\prime \prime} \mathrm{W}\right)$. It is the site that is most representative of the inner zone of the estuary in terms of hydrodynamic conditions and the site with the most marked temperature and salinity fluctuations. The inner zone acts as a true estuary (Negrín 2011) (Fig. 1a).

\section{Field surveys}

We conducted seasonal samplings from autumn 2013 to autumn 2015. Measurements of burrow architecture were performed during the 2013/2014 and 2014/2015 breeding seasons. We selected 2 habitats with visually different vegetation composition and sedimentary and hydrodynamic conditions: a Sarcocornia perennis saltmarsh in the middle intertidal zone and a mudflat in the lower intertidal zone (Fig. 1b).

Burrow density was quantified using quadrats $(0.5 \times 0.5 \mathrm{~m})$, which were randomly placed at each site during low tides, when the area was exposed (10 replicates). The total number of open burrows in each quadrat was counted, and active burrows were identified by the presence of crabs, prints, or removed sediments, which are lighter in color and have different texture (biogenic mounds). Polyester resin and 2 components, accelerator (cobalt octoate solution) and catalyst (methyl ethyl ketone peroxide), were poured into selected active burrows to make burrow casts.

Sediment samples from the adjacent surface were collected to compare sediments from biogenic mounds with sediments from areas without crabs (control) and to determine water and organic matter contents, grain size distribution profiles, and mineralogical composition. Penetrability $\left(\mathrm{N} \cdot \mathrm{cm}^{-2}\right)$ was measured as the pressure needed to compress the spring of a piston that was forced into the sediment to a standard depth (McLachlan and Brown 2006); low pressure values indicated soft sediments and high values indicated firm sediments.

\section{Laboratory measurements and data analysis}

Entrance diameter, tunnel diameter, total length, and total depth were measured from each burrow cast with a digital caliper $( \pm 0.1 \mathrm{~mm})$. Curvature and number of branches and entrances were also recorded for each burrow cast. Only data from complete casts were used for analyses.

Sediment properties were analyzed. Water content was determined as the difference between wet and dry weight (after drying at $60{ }^{\circ} \mathrm{C}$ for $5 \mathrm{~d}$ ). Organic matter content

\section{MATERiales Y MÉTOdos}

\section{Área de estudio}

El estuario de Bahía Blanca, en el suroeste de la provincia de Buenos Aires, tiene una superficie aproximada de $3,000 \mathrm{~km}^{2}$. Es un estuario de llanura costera mesomareal con un régimen de mareas semidiurnas, y se caracteriza por la presencia de islas que están interconectadas por un extenso sistema de canales de marea que es afectado por mareas de hasta $4 \mathrm{~m}$. La entrada de agua dulce al estuario es débil y proviene principalmente de 2 afluentes en la costa norte del interior del sistema (Melo 2004). La salinidad en la porción media del estuario es de aproximadamente 33.98 (media anual), mientras que la zona interna se vuelve hipersalina durante los veranos secos debido a la alta tasa de evaporación (Piccolo et al. 2008). El área de muestreo se ubicó en puerto Cuatreros $\left(38^{\circ} 44^{\prime} 50^{\prime \prime} \mathrm{S}, 62^{\circ} 23^{\prime} 5^{\prime \prime} \mathrm{W}\right)$. Es el sitio más representativo de la zona interna del estuario en términos de condiciones hidrodinámicas y el que presenta las fluctuaciones más marcadas en temperatura y salinidad. La zona interna actúa como un verdadero estuario (Negrín 2011) (Fig. 1a).

\section{Muestreo de campo}

Realizamos muestreos estacionales desde el otoño de 2013 hasta el otoño de 2015. Las mediciones de la arquitectura de las cuevas se realizaron durante las temporadas reproductivas de 2013/2014 y 2014/2015. Se seleccionaron 2 hábitats que visualmente presentaron diferente composición vegetal y distintas condiciones sedimentarias e hidrodinámicas: una marisma de Sarcocornia perennis en la zona intermareal media y una planicie de marea en la zona intermareal inferior (Fig. 1b).

La densidad de cuevas se calculó usando cuadrantes $(0.5 \times 0.5 \mathrm{~m})$ que fueron colocados al azar en cada sitio durante las mareas bajas cuando el área estaba expuesta (10 réplicas). Se contó el número total de cuevas abiertas dentro de cada cuadrante, y se identificaron las cuevas activas por la presencia de cangrejos, impresiones de pinzas o sedimento removido, el cual es de color más claro y textura diferente (montículos biogénicos). Se vertió resina de poliéster más 2 componentes, acelerador (solución de octoato de cobalto) y catalizador (peróxido de metil etil cetona), en las cuevas activas seleccionadas para generar los moldes.

Se tomaron muestras de los sedimentos superficiales adyacentes para comparar los sedimentos de los montículos biogénicos con los sedimentos de áreas sin cangrejos (control) y para determinar el contenido de agua y materia orgánica, la distribución del tamaño de grano y la composición mineralógica. La penetrabilidad $\left(\mathrm{N} \cdot \mathrm{cm}^{-2}\right)$ se midió como la presión necesaria para comprimir el resorte de un pistón que se introdujo en el sedimento a una profundidad estándar (McLachlan y Brown 2006); los valores bajos de presión indicaron 

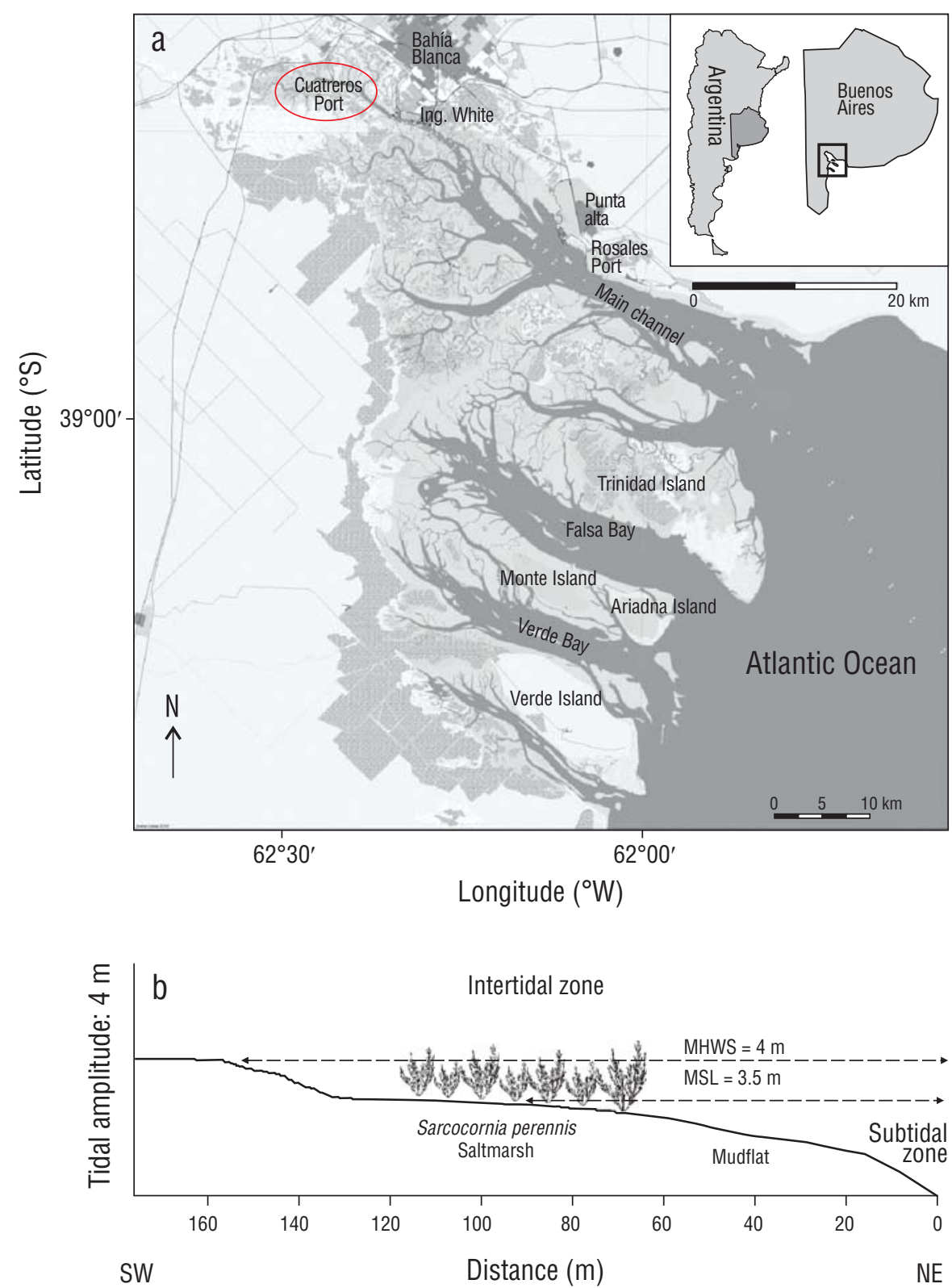

Figure 1. (a) Study area with zoning of the sampling site (red outline). (b) Schematic representation of the sampling site. MHWS, mean high water springs; MSL, mean sea level.

Figura 1. (a) Área de estudio con la zonificación del sitio de muestreo (delineado rojo). (b) Representación esquemática del sitio de muestreo. MHWS, nivel medio de mareas vivas; MSL, nivel medio del mar.

was taken to be the difference between dry and ash-free dry weight (after ignition in a muffle furnace at $500{ }^{\circ} \mathrm{C}$ for $7 \mathrm{~h}$ ).

For grain size analysis, samples were dried to constant weight and organic matter was removed with hydrogen peroxide at 130 volumes, initially cold and then hot $\left(60^{\circ} \mathrm{C}\right)$ until bubbling of organic material had ceased. This methodology was used to avoid interference with the determination of clay content. A laser diffraction particle size analyzer (Malvern Mastersizer 2000) was used to determine particle size distribution (0.2-2000 $\mu \mathrm{m}$ particles). Data were analyzed using sedimentos blandos, mientras que los valores altos indicaron sedimentos firmes.

\section{Mediciones de laboratorio y análisis de datos}

El diámetro de entrada, el diámetro de túnel, la longitud total y la profundidad total de cada molde de cueva fueron medidos con un calibre digital $( \pm 0.1 \mathrm{~mm})$. La curvatura, el número de ramas y el número de entradas de cada molde de cueva también fueron registrados. Solo se usaron los datos de los moldes enteros para los análisis. 
Mastersizer 2000 v5.40 (Malvern Instrument Ltd. 2007) and Gradistat v5.0 (Blott and Pye 2001) software.

To determine the mineralogical composition of sediments, samples were dried to constant weight and then milled using an agate mortar. An X-ray diffraction analysis was performed with a Rigaku D-Max III-C diffractometer with $\mathrm{CuK} \alpha$ radiation and a graphite monochromator at $35 \mathrm{Kv}$ and $15 \mathrm{~mA}$ for the qualitative determination of minerals. The loose grain method was also applied under a magnifying glass using a B2-UMA trinocular Olympus stereomicroscope and computerized image processing programs.

To analyze the differences in mean burrow densities and sediment properties, two-way analyses of variance (ANOVA) were used. If a significant interaction was obtained, the main effects of fixed factors were not considered since this type of interaction indicates lack of independence (Underwood 1997). The null hypothesis of no differences in burrow architectural variables between microhabitats was assessed using one-way ANOVA (Zar 1999). For all statistical analyses, data were previously transformed to comply with the normality and homoscedasticity assumptions. In the case of significant differences with ANOVA, a posteriori multiple-comparison tests (Tukey test, Zar 1999) were used to identify significant differences.

\section{ReSULTS}

The total number of burrows (per square meter) differed significantly between sites $(F=236.78, P<0.001)$ but not between seasons $(F=1.26, P>0.05)$. The effects of interaction between sites and seasons were also not significant (two-way ANOVA interaction: $F=0.19, P>0.05$ ). Maximum was 172 burrows $\cdot \mathrm{m}^{-2}$ in autumn in the mudflat (Fig. 2a). When only the active burrows were considered, differences occurred between sites $(F=55.36, P<0.001)$ and between seasons $(F=6.61, P<0.001)$. The effects of interaction between sites and seasons were also significant (two-way ANOVA interaction: $F=19.47, P<0.001$ ). The highest number of active burrows was found in the mudflat too, but unlike the previous case, significantly higher values were observed in spring and summer. The maximum number of active burrows ( 144 burrows $\cdot \mathrm{m}^{-2}$ ) was recorded in summer in the mudflat, where all the burrows showed signs of activity (Fig. 2b).

Water content of sediments varied significantly between microhabitats $(F=18.90, P<0.001)$. The sediments from the mudflat had higher water content than those from the saltmarsh. The effect of crab bioturbation on sediment condition was also significant $(F=6.71, P<0.05)$; removed sediments had higher water content than control sediments. The effect of this interaction was not significant $(F=0.16, P>0.05)$ (Fig. 3a). Organic matter content did not show significant differences between microhabitats $(F=0.04, P>0.05)$ or between sediment conditions $(F=0.09, P>0.05)$. The effect of the interaction was also not significant $(F=0.10, P>0.05)$
Las propiedades del sedimento fueron analizadas. El contenido de agua fue determinado como la diferencia entre el peso húmedo y el peso seco (después de secar a $60^{\circ} \mathrm{C}$ durante $5 \mathrm{~d}$ ). El contenido de materia orgánica fue determinado como la diferencia entre el peso seco y el peso seco sin cenizas (después de la ignición en un horno de mufla a $500{ }^{\circ} \mathrm{C}$ durante $7 \mathrm{~h}$ ).

Para el análisis granulométrico, las muestras se secaron hasta obtener un peso constante y se eliminó la materia orgánica con peróxido de hidrógeno a 130 volúmenes, inicialmente en frío y luego en caliente $\left(60^{\circ} \mathrm{C}\right)$ hasta que cesó el burbujeo del material orgánico. Esta metodología se usó para evitar interferencias en la determinación del contenido de arcilla. Se utilizó un analizador de partículas por difracción láser (Malvern Mastersizer 2000) para determinar la distribución del tamaño de las partículas (partículas de $0.2-2,000 \mu \mathrm{m}$ ). Los datos se analizaron utilizando el software Mastersizer 2000 v5.40 (Malvern Instrument Ltd. 2007) y Gradistat v5.0 (Blott y Pye 2001).

Para determinar la composición mineralógica de los sedimentos, las muestras se secaron hasta obtener un peso constante y luego se molieron en un mortero de ágata. El análisis de difracción de rayos $\mathrm{X}$ se realizó con un difractómetro Rigaku D-Max III-C con radiación CuKa y un monocromador de grafito con $35 \mathrm{Kv}$ y $15 \mathrm{~mA}$ para la determinación cualitativa de los minerales. También se utilizó el método de grano suelto bajo una lupa, con un estereomicroscopio Olympus trinocular B2-UMA y programas computarizados de procesamiento de imágenes.

Para analizar las diferencias en las densidades medias de las cuevas y las propiedades de los sedimentos, se utilizaron análisis de varianza (ANDEVA) de 2 vías. Cuando una interacción fue significativa, no se consideraron los efectos principales de los factores fijos ya que este tipo de interacciones indica dependencia (Underwood 1997). La hipótesis nula de que no existen diferencias en las variables de la arquitectura de las cuevas entre microhábitats se evaluó utilizando el ANDEVA de una vía (Zar 1999). Para todos los análisis estadísticos, los datos se transformaron previamente para cumplir con los supuestos de normalidad y homocedasticidad. En el caso de las diferencias significativas con el ANDEVA, se usaron pruebas de comparación múltiple a posteriori (prueba de Tukey, Zar 1999) para identificar diferencias significativas.

\section{Resultados}

El número total de cuevas (por metro cuadrado) mostró diferencias significativas entre los sitios $(F=236.78, P<$ $0.001)$, pero no entre las estaciones $(F=1.26, P>0.05)$. Los efectos de la interacción entre los sitios y las estaciones tampoco fueron significativos (interacción ANDEVA de 2 vías: $F=0.19, P>0.05$ ). La densidad máxima fue de 172 cuevas $\cdot \mathrm{m}^{-2}$ en otoño en la planicie de marea (Fig. 2a). Cuando se consideraron solo las cuevas activas, se encontraron diferencias entre los sitios $(F=55.36, P<0.001)$ y entre las estaciones $(F=6.61, P<0.001)$. Los efectos de la 
(Fig. 3b). When sediment penetrability was analyzed, significant differences were found between microhabitats $(F=7.89, P<0.05)$ and sediment conditions $(F=9.44, P<$ $0.05)$. Sediments from the mudflat had higher penetrability (lower pressure to penetrate the substrate) than sediments from the saltmarsh. Sediments removed from burrows had higher penetrability than control sediments. The interaction effect of these factors was not significant $(F=0.53, P>0.05)$ (Fig. 3c).

The grain size distribution profiles and mineralogical analysis did not show significant differences between sediments from the saltmarsh and sediments from the mudflat. These sediments were composed of thick and very thick limes and very fine sands. Most of the measurements were characterized by one mode between approximately 6 and $60 \mu \mathrm{m}$, corresponding to silt particles (Fig. 4). The mineralogical composition of the intertidal sediments was represented mostly by quartz, feldspar, calcite, and halite (salt), and to a lesser extent by clays such as illite and montmorillonite (Fig. 5). No seasonal variations were observed.

Biogenic mounds did not have a specific orientation. No general pattern was observed with their position in relation to the mouth of the burrow or to the location relative to the subtidal zone. The diameters of burrow entrances measured in the field showed significant differences between microhabitats $(F=29.71, P<0.01)$. Burrow entrances from the mudflat were found to be significantly narrower, compared to the burrows from the saltmarsh (Fig. 6).

From the casts obtained during field sampling, we observed that burrows had both simple and complex morphologies, ranging from vertical tunnels with a single entrance to complex interconnected tunnels with multiple interacción entre los sitios y las estaciones también fueron significativos (interacción ANDEVA de 2 vías: $F=19.47$, $P<0.001)$. El mayor número de cuevas activas también se encontró en la planicie de marea, pero a diferencia del caso anterior, se observaron valores significativamente más altos en primavera y verano. El número máximo de cuevas activas (144 cuevas $\cdot \mathrm{m}^{-2}$ ) se registró en verano en la planicie de marea, donde todas las cuevas mostraron signos de acti$\operatorname{vidad}$ (Fig. 2b).

El contenido de agua de los sedimentos varió significativamente entre los microhábitats $(F=18.90, P<0.001)$. Los sedimentos de la planicie de marea presentaron un mayor contenido de agua que los de la marisma. El efecto de la bioturbación del cangrejo sobre la condición del sedimento también fue significativo $(F=6.71, P<0.05)$; el sedimento removido presentó un mayor contenido de agua que el control. El efecto de esta interacción no fue significativo $(F=0.16, P>0.05)$ (Fig. 3a). El contenido de materia orgánica no mostró diferencias significativas entre los microhábitats $(F=0.04, P>0.05)$ o entre las condiciones del sedimento $(F=0.09, P>0.05)$. El efecto de la interacción tampoco fue significativo $(F=0.10, P>0.05)$ (Fig. 3b). Cuando se analizó la penetrabilidad del sedimento, se encontraron diferencias significativas entre los microhábitats $(F=7.89, P<0.05)$ y entre las condiciones del sedimento $(F=9.44, P<0.05)$. Los sedimentos de la planicie de marea mostraron una mayor penetrabilidad (menor presión para penetrar el sustrato) que los sedimentos de la marisma. Los sedimentos removidos de las cuevas presentaron mayor penetrabilidad que los sedimentos control. El efecto de interacción de estos factores no fue significativo $(F=0.53, P>0.05)($ Fig. 3c).
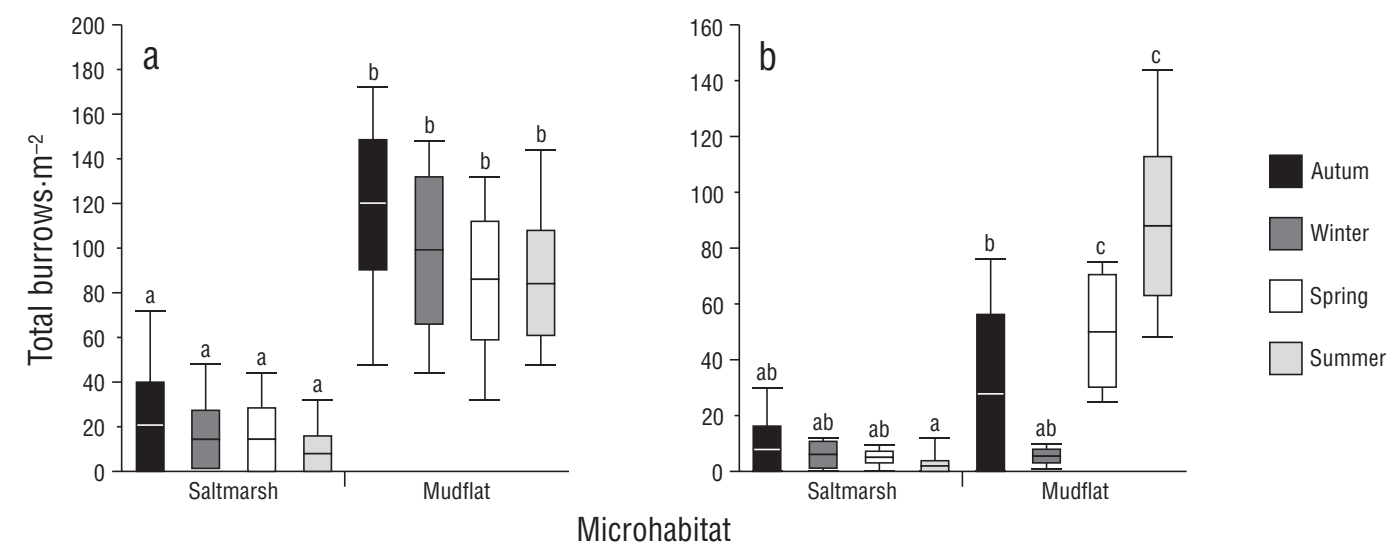

Figure 2. Density of Neohelice granulata burrows. Number of total burrows (a) and number of active burrows (b) in 2 microhabitats throughout the seasons. Lines inside boxes are median values, box limits are standard errors of the mean, and error bars represent non-outlier ranges. Different letters above the boxes denote significant differences between groups of data (Tukey test after ANOVA, $P<0.05$ ).

Figura 2. Densidad de las cuevas de Neohelice granulata. Número total de cuevas (a) y número de cuevas activas (b) en 2 microhábitats a través de las estaciones. Las líneas dentro de las cajas son los valores de la mediana, los límites de las cajas son los errores estándar del promedio y las barras de error representan el rango de los valores no anómalos. Las letras sobre las cajas indican diferencias significativas entre los grupos de datos (prueba de Tukey después del análisis de varianza, $P<0.05$ ). 

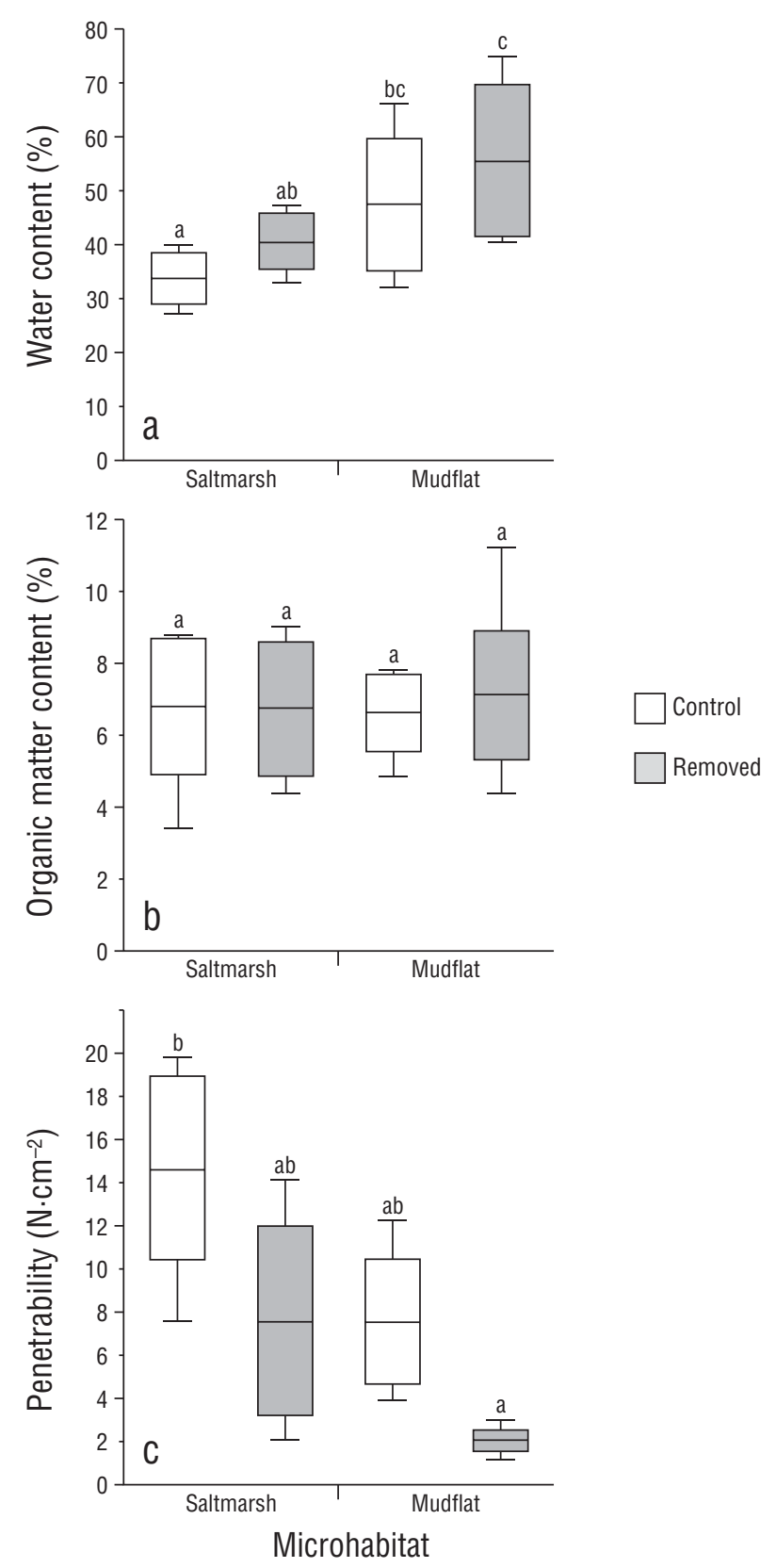

Figure 3. Sediment properties in 2 microhabitats: water content (a), organic matter content (b), and penetrability (c) in control sediments and sediments removed by crabs. Lines inside boxes are median values, box limits are standard errors of the mean, and error bars represent non-outlier ranges. Different letters above the boxes denote significant differences between groups of data (Tukey test after ANOVA, $P<0.05$ ).

Figura 3. Propiedades de los sedimentos de 2 microhábitats: contenido de agua (a), contenido de materia orgánica (b) y penetrabilidad (c) en el sedimento control y el sedimento removido por cangrejos. Las líneas dentro de las cajas son los valores de la mediana, los límites de las cajas son los errores estándar del promedio y las barras de error representan los rangos de los valores no anómalos. Las letras sobre las cajas indican diferencias significativas entre los grupos de datos (prueba de Tukey después del análisis de varianza, $P<0.05)$.
Los perfiles de distribución de tamaños de grano y el análisis mineralógico no mostraron diferencias significativas entre los sedimentos de la marisma y los sedimentos la planicie de marea. Estos sedimentos estuvieron constituidos por limos gruesos y muy gruesos y arenas muy finas. La mayoría de las mediciones se caracterizaron por una moda entre aproximadamente 6 y $60 \mu \mathrm{m}$, correspondiente a las partículas de limo (Fig. 4). La composición mineralógica de los sedimentos intermareales estuvo representada principalmente por cuarzo, feldespato, calcita y halita (sal), y en menor medida por arcillas como illita y montmorillonita (Fig. 5). No se observaron variaciones estacionales.

Los montículos biogénicos no presentaron una orientación específica. No se observó un patrón general en cuanto a su posición en relación con la boca de las cuevas o la ubicación relativa a la zona submareal. Los diámetros de las entradas de las cuevas medidas en campo mostraron diferencias significativas entre los microhábitats $(F=29.71, P<0.01)$. Las entradas de las cuevas de la planicie de marea fueron significativamente más estrechas, en comparación con las cuevas de la marisma (Fig. 6).

A partir de los moldes de las cuevas obtenidos durante el muestreo de campo, pudimos observar que las cuevas presentaron morfologías simples y complejas, ya que variaron desde túneles verticales con una sola entrada hasta túneles complejos e interconectados con múltiples entradas. Los diámetros de las entradas no presentaron diferencias significativas entre los microhábitats $(F=0.53, P>0.05)$, como así tampoco los diámetros de los túneles $(F=0.06, P>0.05)$. En cuanto a la variable longitud, encontramos diferencias significativas entre los microhábitats $(F=6.88, P<0.05)$. También encontramos diferencias significativas en la profundidad de las cuevas entre los microhábitats $(F=5.85, P<0.05)$. Los moldes de las cuevas de la marisma fueron significativamente más largos y más profundos que los de la planicie de marea (Fig. 7). La relación entre la profundidad del túnel y el diámetro de la entrada (relación $>1$ ) nos permitió clasificar a todos los moldes como tubulares. Se encontraron cámaras dentro de las cuevas de las marismas. Estas cámaras se registraron solo en los túneles, no así en las entradas de las cuevas.

\section{Discusión}

La densidad de $N$. granulata disminuye hacia la zona intermareal superior, como lo expresaron Bortolus e Iribarne (1999). Sin embargo, la colonización de la marisma alta por $N$. granulata se ve facilitada por la presencia de plantas. Sarcocornia perennis es un buen colonizador de zonas intermareales altas y salinas. Genera un área de sombreado que permite que el sedimento permanezca más húmedo, por lo tanto, más suave y más propenso a ser excavado. También amortigua diversos factores ambientales, como la desecación y las altas temperaturas superficiales (Bortolus et al. 2002).

La densidad máxima de cuevas totales de $N$. granulata registrada en este estudio fue de 72 cuevas $\cdot \mathrm{m}^{-2}$ en la marisma 


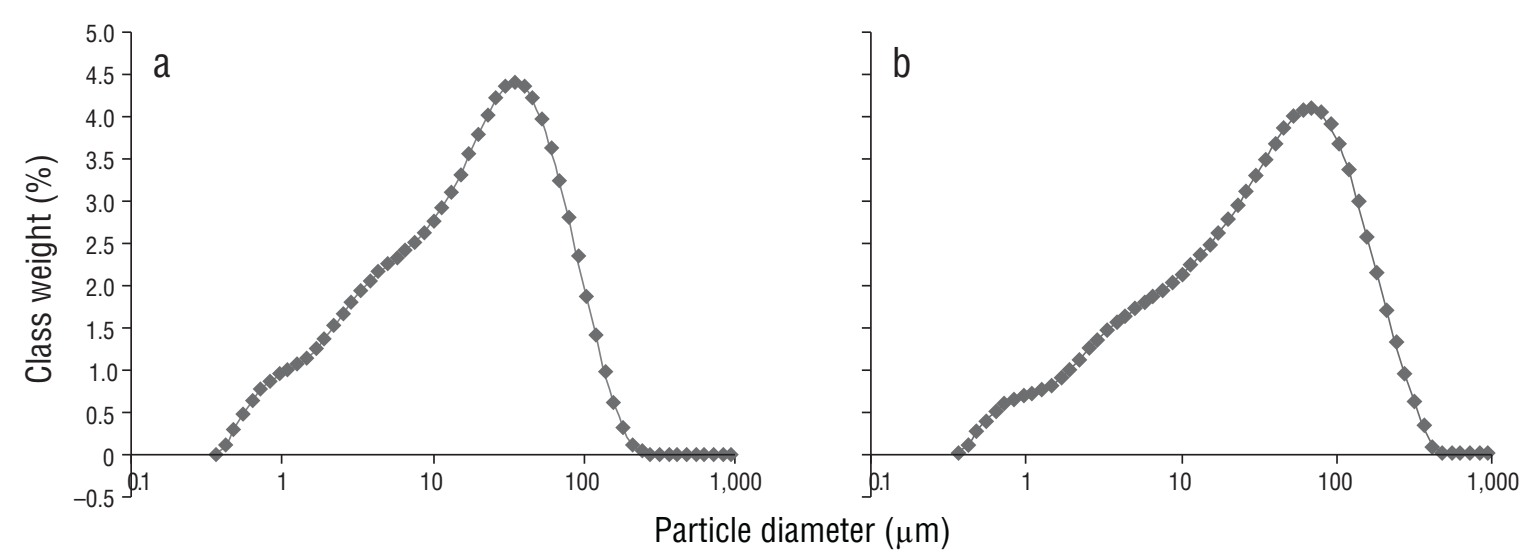

Figure 4. Grain size distribution profiles. (a) Saltmarsh. (b) Mudflat.

Figura 4. Perfiles de distribución de tamaños de grano. (a) Marisma. (b) Planicie de marea.

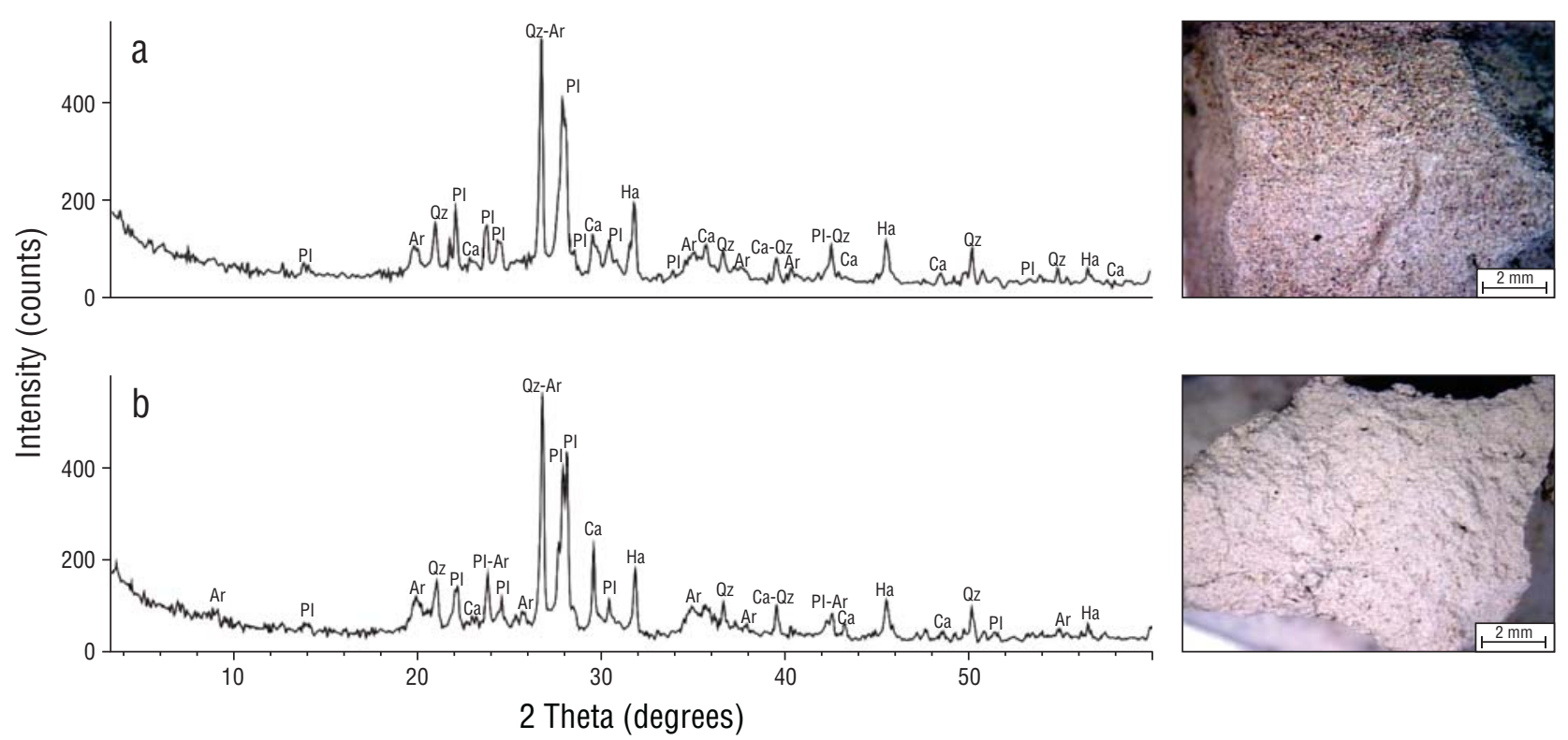

Figure 5. X-ray diffraction analysis. (a) Saltmarsh. (b) Mudflat. Qz, quartz; Pl, feldspar; Ar, clay; Ha, halite; Ca, calcite.

Figura 5. Análisis de difracción de rayos X. (a) Marisma. (b) Planicie de marea. Qz, cuarzo; Pl, feldespato; Ar, arcilla; Ha, halita; Ca, calcita.

entrances. Entrance diameters did not show significant differences between the microhabitats $(F=0.53, P>0.05)$, nor did the tunnel diameters $(F=0.06, P>0.05)$. While for the length variable, significant differences occurred between microhabitats $(F=6.88, P<0.05)$. We also found significant differences in burrow depth between microhabitats $(F=5.85, P<$ $0.05)$. The casts from the saltmarsh were significantly longer and deeper than those from the mudflat (Fig. 7). The relationship between the depth of the tunnel and the diameter of the entrance (ratio $>1$ ) allowed all casts to be classified as tubular. Chambers were found inside burrows from the saltmarsh. These chambers were recorded only within the tunnels, not at the entrances of the burrows. y 172 cuevas $\cdot \mathrm{m}^{-2}$ en la planicie de marea. Estos resultados son comparativamente similares, y algunas veces superiores, a los registrados por otros autores para la costa argentina. Escapa et al. (2008) y Montemayor et al. (2011) encontraron un promedio de 64.8 cuevas $\cdot \mathrm{m}^{-2}$ en las marismas y un máximo de 75 cuevas $\cdot \mathrm{m}^{-2}$ en las planicies de marea en la sección interna del estuario de Bahía Blanca. En la laguna Mar Chiquita, las cuevas pueden alcanzar una densidad máxima de 60 cuevas $\cdot \mathrm{m}^{-2}$ en las marismas y 100 cuevas $\cdot \mathrm{m}^{-2}$ en las planicies de marea (Iribarne et al. 1997, Bortolus et al. 2002). En contraste, en los humedales de Santa Lucía, Uruguay, un máximo de 95 cuevas $\cdot \mathrm{m}^{-2}$ fue reportado para las marismas (Merentiel-Ferreyra 2014). 


\section{Discussion}

Neohelice graulata density decreases towards the upper intertidal zone, as noted by Bortolus and Iribarne (1999). However the colonization of the high marsh by $N$. granulata is facilitated by the presence of plants. Sarcocornia perennis is a good colonizer of high and saline intertidal areas. It generates a shading area allowing the sediment to stay wetter, therefore softer and more liable to being excavated. It also dampens various environmental factors, such as desiccation and high surface temperatures (Bortolus et al. 2002).

The maximum density of total $N$. granulata burrows recorded in this study was 72 burrows $\cdot \mathrm{m}^{-2}$ for the saltmarsh and 172 burrows $\cdot \mathrm{m}^{-2}$ for the mudflat. These densities are comparatively similar, and sometimes higher, to those reported by other authors for the Argentinean coast. An average of 64.8 burrows $\cdot \mathrm{m}^{-2}$ in saltmarshes and a maximum of 75 burrows $\cdot \mathrm{m}^{-2}$ in mudflat areas were found by Escapa et al. (2008) and Montemayor et al. (2011) in the internal section of the Bahía Blanca Estuary. In the Mar Chiquita Lagoon, burrows can reach a maximum density of 60 burrows $\cdot \mathrm{m}^{-2}$ in saltmarshes and 100 burrows $\cdot \mathrm{m}^{-2}$ in mudflats (Iribarne et al. 1997, Bortolus et al. 2002). By contrast, in the wetlands of Santa Lucía, Uruguay, a maximum of 95 burrows $\cdot \mathrm{m}^{-2}$ were recorded in saltmarshes (Merentiel-Ferreyra 2014).

A higher density of burrows in autumn might be related to the molting phenomenon. According to Luppi et al. (2013), $N$. granulata is almost inactive on the intertidal surface of the Mar Chiquita Lagoon and Bahía Blanca Estuary during this season, regardless of the daily variation in environmental conditions. So the burrows increase in number and their entrances are closed up with mud deposits. Crabs remain inside the burrows during the pre- and post-molt phases, suggesting a rather synchronized molting season. Burrows are the best refuge for the species during this delicate stage.

Total and active burrow densities showed high variation throughout the year in the mudflat, possibly one of the most dynamic areas in the intertidal zone. In this microhabitat we found the highest densities of active burrows in the summer, compared to the rest of the year. In addition, ovigerous females have been found only in this microhabitat; this could be interpreted as a reproductive advantage for the species. Settling in areas near the coastline during the summer months would allow ovigerous females to better oxygenate their eggs and readily release their larvae (Anger et al. 1994).

The physical properties of the sediments from the mudflat (e.g., the high water content and penetrability) might be correlated with their proximity to the subtidal zone, which is usually saturated with water. Sediments removed by crab activity had higher water content and penetrability than control sediments; this is in agreement with Botto and Iribarne (2000) and Escapa et al. (2007), who indicated that bioturbation increases the values of these parameters. No significant
Una mayor densidad de cuevas durante el otoño podría estar relacionada con el fenómeno de la muda. De acuerdo con Luppi et al. (2013), N. granulata se mantiene casi inactivo en la superficie de la zona intermareal de la laguna Mar Chiquita y el estuario de Bahía Blanca en esa estación, independientemente de la variación diaria en las condiciones ambientales. Por lo tanto, las cuevas aumentan en número y sus entradas se cierran con depósitos de barro. Los cangrejos permanecen dentro de las cuevas antes y después de la muda, lo que sugiere una temporada de muda sincronizada. Las cuevas son el mejor refugio para que la especie afronte esta delicada etapa.

Las densidades de las cuevas totales y activas variaron mucho a lo largo del año en la planicie de marea, posiblemente una de las áreas más dinámicas de la zona intermareal. En este microhábitat encontramos las densidades más altas de cuevas activas en el verano, en comparación con el resto del año. Además, las hembras ovígeras se han encontrado solo en este microhábitat; esto podría interpretarse como una ventaja reproductiva para la especie. El establecerse en áreas cercanas a la costa durante los meses de verano permitiría a las hembras ovígeras oxigenar mejor sus huevos y liberar las larvas con más facilidad (Anger et al. 1994).

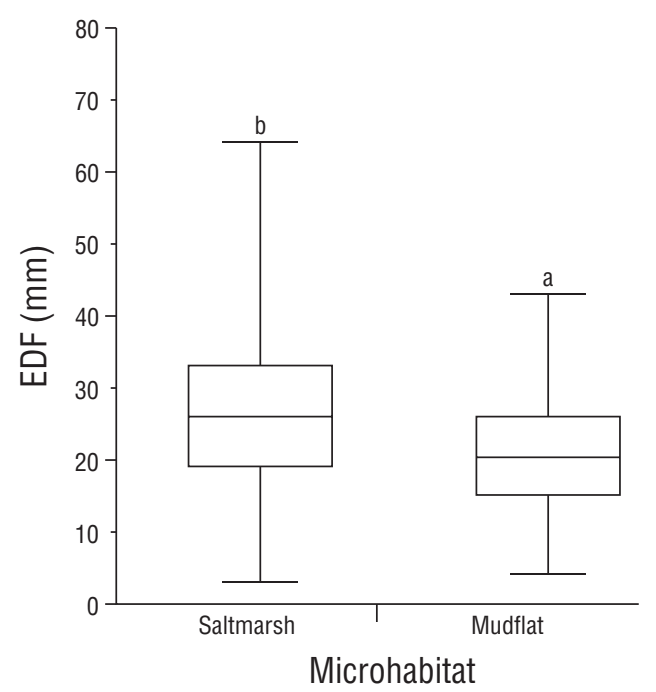

Figure 6. Entrance diameters of burrows measured in the field (EDF) in 2 microhabitats. Lines inside boxes are median values, box limits are standard errors of the mean, and error bars represent non-outlier ranges. Different letters above the boxes denote significant differences between groups of data (Tukey test after ANOVA, $P<0.05)$.

Figura 6. Diámetros medidos en campo (EDF) de las entradas de las cuevas de 2 microhábitats. Las líneas dentro de las cajas son los valores de la mediana, los límites de las cajas son los errores estándar del promedio y las barras de error representan los rangos de los valores no anómalos. Las letras sobre las cajas indican diferencias significativas entre los grupos de datos (prueba de Tukey después del análisis de varianza, $P<0.05$ ). 

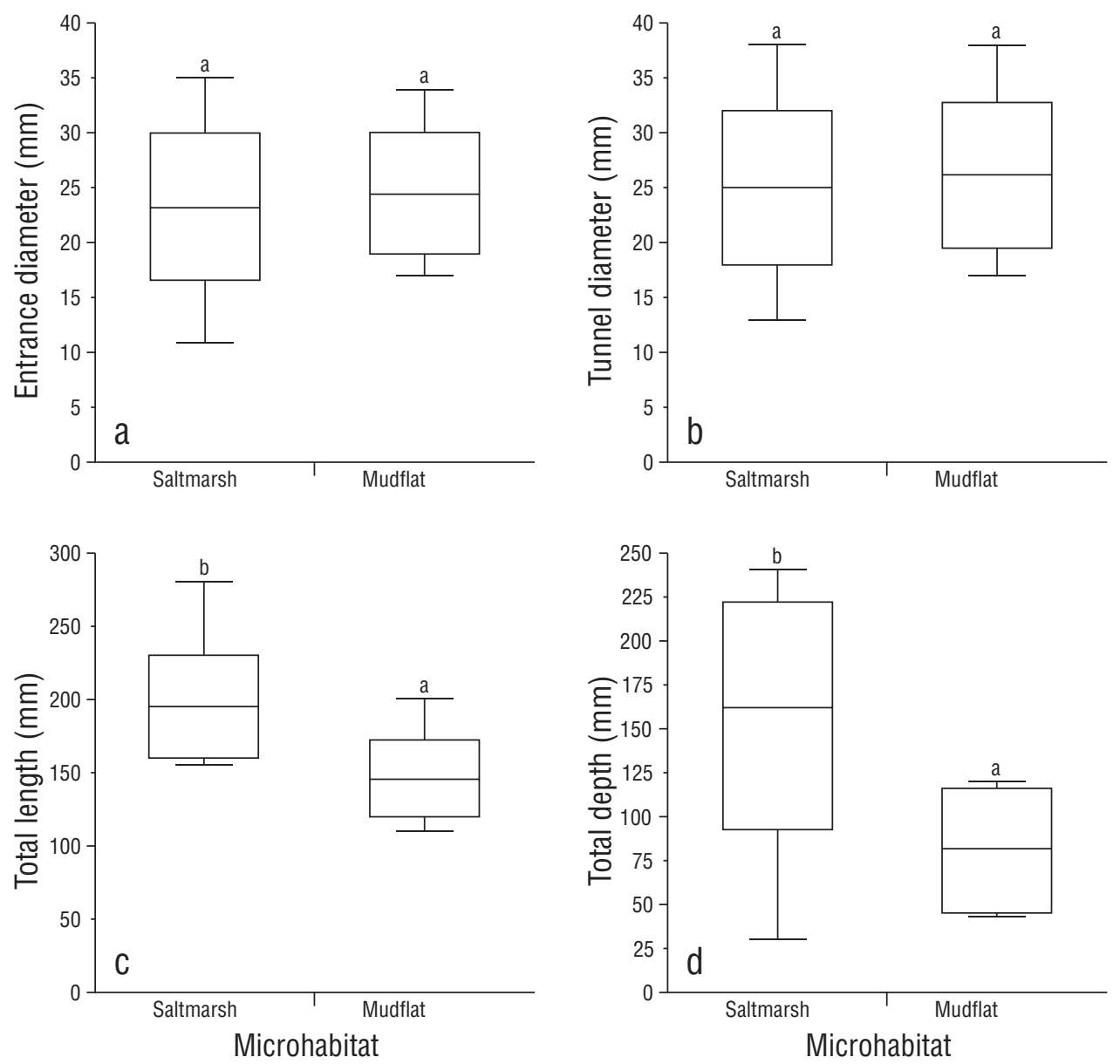

Figure 7. Parameters measured in burrow casts. (a) Entrance diameter. (b) Tunnel diameter. (c) Total length. (d) Total depth. Lines inside boxes are median values, box limits are standard errors of the mean, and error bars represent non-outlier ranges. Different letters above the boxes denote significant differences between groups of data (Tukey test after ANOVA, $P<0.05$ ).

Figura 7. Parámetros medidos en los moldes de las cuevas. (a) Diámetro de las entrada. (b) Diámetro de tunel. (c) Longitud total. (d) Profundidad total. Las líneas dentro de las cajas son los valores de la mediana, los límites de las cajas son los errores estándar del promedio y las barras de error representan los rangos de los valores no anómalos. Las letras sobre las cajas indican diferencias significativas entre los grupos de datos (prueba de Tukey después del análisis de varianza, $P<0.05$ ).

differences were found in the contents of organic matter between sediments, because remixing of sediments by the species kept organic matter homogeneously dispersed in the sedimentary column (Bortolus and Iribarne 1999, Gutiérrez et al. 2006).

The predominant fractions in the grain size distribution profile corresponded to the medium size (medium to fine sands) and the fine size (silts and clays). Homogeneous sediments indicate a low-energy environment. Tidal channels in low-energy environments branch out and leave wide, shallow flood plains, and energy in these areas consequently decreases (Gelós et al. 2004). No significant variations were found in the mineralogical composition of sediments, only in the proportion of minerals present in the sediments from the 2 microhabitats. This study constitutes the first mineralogical results for 2 intertidal zones from the Bahía Blanca Estuary that had not been previously studied.
Las propiedades físicas de los sedimentos de la planicie de marea (e.g., el alto contenido de agua y la gran penetrabilidad) podrían correlacionarse con su proximidad a la zona submareal, que generalmente se encuentra saturada de agua. Los sedimentos removidos por la bioturbación de los cangrejos tuvieron un mayor porcentaje de agua y penetrabilidad que los sedimentos control, concordando con Botto e Iribarne (2000) y Escapa et al. (2007), quienes afirmaron que la bioturbación aumenta los valores de estos parámetros. No se encontraron diferencias significativas en los contenidos de materia orgánica entre los sedimentos, ya que esta estaba homogéneamente dispersa en la columna sedimentaria debido a la mezcla de sedimentos generada por la especie (Bortolus e Iribarne 1999, Gutiérrez et al. 2006).

Las fracciones predominantes en la distribución del tamaño de grano fueron aquellas correspondientes al tamaño mediano (arenas medianas a finas) y al tamaño fino (limos 
Burrows in mudflats had a weak structure. They sometimes collapsed when the polyester resin was poured into them. In some cases burrows entrance diameters were overestimated because of this. This is the reason we found differences in the diameters of the burrow entrances from the mudflats when we used both methodologies. However, burrows in the upper intertidal zone were well structured. We believe that the diameters measured in the field are more accurate estimates of the actual dimensions of the entrances than those measured from the casts.

In our study site we found burrows with ramifications and multiple entrances, and this was directly associated with the type of substrate on which the burrows were built. Several experiments with Austrohelice crassa crabs showed that the burrows from sandy sites were unique and simple, while those at sites with a higher proportion of the clay-silt fraction were more complex in structure, with multiple vertical ramifications that were horizontally interconnected through tunnels (Morrisey et al. 1999). These multiple entrances may provide easy access to the shelter, especially in un-vegetated mudflats. In addition, there is evidence suggesting that a larger number of burrow entrances in intertidal mudflats increase the ability to entrap organic particles that may serve as food (Iribarne et al. 1997, Botto et al. 2006). This phenomenon was also observed for species of the genus Uca (Genoni 1991, Qureshi and Saher 2012) and in our study would be supported by the high densities of burrows found in the mudflat.

Burrows from the saltmarsh were longer and deeper than those from the mudflat near the subtidal zone for the internal zone of the Bahía Blanca Estuary. Crabs could change the depth of their burrows in relation to water table depth, to reach groundwater levels. In this way, the burrows would contain water throughout the tide cycle, an adaptation to maintain the humidity in the high areas of the marshes (Iribarne et al. 1997, Escapa et al. 2007).

The burrows with chambers are not mentioned in the bibliography for the Bahía Blanca Estuary. However, copulation is likely to occur not only on the surface but also within the burrows, as was found for the Grapsoidea superfamily by Brockerhoff and McLay (2005). In contrast, burrows from mudflats could have chambers in their tunnels, although they would not be as conspicuous as the ones described by these authors, since the value that was used to detect them $(18 \mathrm{~mm}$, Sal-Moyano et al. 2012) could be a very high parameter for this microhabitat.

Our observations and field experiments showed that burrow features and burrowing activity of $N$. granulata differed between study sites, according to biotic and abiotic factors, indicating that this species has an adaptive burrowing behavior. Moreover, in this study we demonstrated that burrowing activity affects some sediment properties. It is important to highlight that in environments with intermediate levels of energy (such as Bahía Blanca Estuary), burrowing organisms play a very significant role. They destabilize the y arcillas). Los sedimento homogéneos indican un ambiente de baja energía. Los canales de marea en ambientes de baja energía se ramifican y crean amplias llanuras de inundación de poca profundidad; como consecuencia, la energía disminuye en estas zonas (Gelós et al. 2004). No se encontraron variaciones significativas en la composición mineralógica de los sedimentos, sólo en la proporción de minerales presentes en los sedimentos de los 2 microhábitats. Este estudio constituye los primeros resultados mineralógicos de 2 zonas intermareales del estuario de Bahía Blanca que no se habían estudiado previamente.

Las cuevas de la planicie de marea tuvieron una estructura débil. Algunas veces se colapsaron al verter la resina de poliéster dentro de ellas. En algunos casos, esto ocasionó que se sobreestimaran los diámetros de las entradas de las cuevas. Por esta razón, encontramos diferencias en los diámetros de las entradas de las cuevas de la planicie de marea cuando usamos ambas metodologías. Sin embargo, las cuevas de la zona intermareal superior estaban bien estructuradas. Creemos que los diámetros medidos en campo son una estimación más precisa del diámetro real de las entradas que aquellos medidos de los moldes.

En nuestro sitio de estudio encontramos cuevas con múltiples ramificaciones y entradas, y esto estuvo directamente relacionado con el tipo de sustrato sobre el cual se construyeron las cuevas. Varios experimentos con cangrejos Austrohelice crassa demostraron que las cuevas de los sitios arenosos eran únicas y simples, mientras que aquellas en sitios con mayor proporción de la fracción arcilla-limo eran de estructura más compleja, con múltiples ramificaciones verticales y túneles interconectados horizontalmente (Morrisey et al. 1999). Estas entradas múltiples podrían proporcionar un acceso fácil al refugio, especialmente en planicies de marea donde no hay vegetación. Además, hay evidencia que sugiere que un mayor número de entradas de cuevas en las planicies de marea aumenta la capacidad de atrapar partículas orgánicas que pueden servir como alimento (Iribarne et al. 1997, Botto et al. 2006). Este fenómeno también se observó en especies del género Uca (Genoni 1991, Qureshi y Saher 2012) y en nuestro estudio estaría respaldado por las altas densidades de cuevas encontradas en la planicie de marea.

Las cuevas de la marisma fueron más largas y más profundas que las de la planicie de marea cercana a la zona submareal para la zona interna del estuario de Bahía Blanca. Los cangrejos podrían cambiar la profundidad de sus cuevas en relación con la profundidad de la capa freática, para alcanzar niveles de agua subterránea. De esta forma, las cuevas contendrían agua durante todo el ciclo de la marea, una adaptación para mantener la humedad en las zonas altas de las marismas (Iribarne et al. 1997, Escapa et al. 2007).

Las cuevas con cámaras no se encuentran citadas en la bibliografía del estuario de Bahía Blanca. Sin embargo, es probable que la cópula ocurra no solo en la superficie sino también dentro de las cuevas, como fue evidenciado para la superfamilia Grapsoidea por Brockerhoff y McLay 
substrate, making fresh material available to tidal currents and waves, which ultimately increases the load of suspended sediments within the environment. This favors the bioavailability of sediment in the water column, particularly when there are high densities of bioturbators as in mudflats. This may have important implications for the functioning of estuarine ecosystems.

\section{ACKNOWLedgments}

This project was supported by a Doctoral scholarship from the National Council of Scientific and Technical Research (CONICET, Argentina). We thank Universidad Nacional del Sur (UNS) and Commission of Scientific Investigations (CIC, Buenos Aires). This work is part of a doctoral thesis by SA at UNS. We want to thank Paula D Pratolongo and Jorge O Pierini for their contribution in field measurements of burrow architecture and Perla $\mathrm{H}$ Angeletti for her advice and helpful comments on the manuscript.

\section{REFERENCES}

Anger K, Spivak E, Bas C, Ismael D, Luppi T. 1994. Hatching rhythms and dispersion of decapod crustacean larvae in a brackish coastal lagoon in Argentina. Helgolander Meeresun. 48(4): 445-466.

https://doi.org/10.1007/bf02366257

Bas C, Lancia JP, Luppi T, Méndez-Casariego A, Kittlein M, Spivak E. 2014. Influence of tidal regime, diurnal phase, habitat and season on feeding of an intertidal crab. Mar. Ecol. 35(3): 319-331. https://doi.org/10.1111/maec.12083

Blott SJ, Pye K. 2001. GRADISTAT: a grain size distribution and statistics package for the analysis of unconsolidated sediments. Earth Surf. Processes Landforms 26(11): 1237-1248. https://doi.org/10.1002/esp.261

Bortolus A, Iribarne O. 1999. Effects of the SW Atlantic burrowing crab Chasmagnathus granulata on a Spartina salt marsh. Mar. Ecol. Prog. Ser. 178: 79-88. https://doi.org/10.3354/meps178079

Bortolus A, Schwindt E, Iribarne O. 2002. Positive plant-animal interactions in the high marsh of an Argentinean coastal lagoon. Ecology 83(3): 733-742. https://doi.org/10.2307/3071877

Botto F, Iribarne O. 2000. Contrasting effects of two burrowing crabs (Chasmagnathus granulata and Uca uruguayensis) on sediment composition and transport in estuarine environments. Estuar. Coast. Shelf Sci. 51(2): 141-151. https://doi.org/10.1006/ecss.2000.0642

Botto F, Iribarne O, Gutierrez J, Bava J, Gagliardini A, Valiela I. 2006. Ecological importance of passive deposition of organic matter into burrows of the SW Atlantic crab Chasmagnathus granulatus. Mar. Ecol.: Prog. Ser. 312: 201-210. https://doi.org/10.3354/meps312201

Brockerhoff AM, McLay CL. 2005. Mating behaviour, female receptivity and male-male competition in the intertidal crab Hemigrapsus sexdentatus (Brachyura: Grapsidae). Mar. Ecol. Prog. Ser. 290: 179-191. https://doi.org/10.3354/meps290179

Escapa M, Perillo GME, Iribarne O. 2008. Sediment dynamics modulated by burrowing crab activities in contrasting SW
(2005). En contraste, las cuevas de las planicies de mareas podrían presentar cámaras en sus túneles, aunque no serían tan conspicuas como las descritas por estos autores, ya que el valor que se utilizó para detectarlas $(18 \mathrm{~mm}$, Sal Moyano et al. 2012) podría ser un parámetro muy alto para este microhábitat.

Nuestras observaciones y experimentos de campo mostraron que las características de las cuevas y la actividad excavadora de $N$. granulata difieren entre los sitios de estudio, de acuerdo con factores bióticos y abióticos, lo que indica que esta especie tiene un comportamiento excavador adaptativo. Además, en este estudio demostramos que la actividad excavadora afecta algunas propiedades del sedimento. Es importante resaltar que en ambientes con niveles intermedios de energía (como es el caso del estuario de Bahía Blanca), los organismos excavadores tienen una función muy importante. Estos desestabilizan el sustrato y, por tanto, hacen que el material fresco esté disponible para las corrientes de marea y las olas, lo que en última instancia aumenta la carga de sedimentos en suspensión en el ambiente. Esto favorece la biodisponibilidad de sedimentos en la columna de agua, especialmente cuando hay grandes densidades de bioturbadores como en las planicies de marea. Esto puede tener implicaciones importantes para el funcionamiento de los ecosistemas estuarinos.

\section{Agradecimientos}

Este proyecto fue apoyado por una beca de doctorado del Consejo Nacional de Investigaciones Científicas y Técnicas (CONICET, Argentina). Agradecemos a la Universidad Nacional del Sur (UNS) y a la Comisión de Investigaciones Científicas (CIC, Buenos Aires). Este trabajo es parte de la tesis doctoral de SA en la UNS. Queremos agradecer a Paula D Pratolongo y Jorge O Pierini su contribución en los muestreos de campo de la arquitectura de las cuevas y a Perla $\mathrm{H}$ Angeletti sus consejos y útiles comentarios en el manuscrito.

Atlantic intertidal habitats. Estuar. Coast. Shelf Sci. 80(3): 365-373.

https://doi.org/10.1016/j.ecss.2008.08.020

Escapa M, Minkoff DR, Perillo GME, Iribarne O. 2007. Direct and indirect effects of burrowing crab Chasmagnathus granulatus activities on erosion of southwest Atlantic Sarcocorniadominated marshes. Limnol. Oceanogr. 52(6): 2340-2349. https://doi.org/10.4319/1o.2007.52.6.2340

Gelós EM, Marcos AO, Spagnolo JO, Schillizi RA. 2004. Textura y mineralogía de los sedimentos. In: Píccolo MC, Hoffmeyer MS (eds.), Ecosistema del Estuario de Bahía Blanca. Instituto Argentino de Oceanografía, Bahía Blanca, Argentina, pp. 43-50.

Genoni GP. 1991. Increased burrowing by fiddler crabs Uca rapax (Smith) (Decapoda: Ocypodidae) in response to low food supply. J. Exp. Mar. Biol. Ecol. 147(2): 267-285.

https://doi.org/10.1016/0022-0981(91)90186-Z 
Gutiérrez JL, Jones CG, Groffman PM, Findlay SEG, Iribarne OO, Ribeiro PD, Bruschetti CM. 2006. The contribution of crab burrow excavation to carbon availability in surficial salt-marsh sediments. Ecosystems 9(4): 647-658. https://doi.org/10.1007/s10021-006-0135-9

Iribarne O, Bortolus A, Botto F. 1997. Between-habitat differences in burrow characteristics and trophic modes in the southwestern Atlantic burrowing crab Chasmagnathus granulata. Mar. Ecol.: Prog. Ser. 155: 137- 145. https://doi.org/10.3354/meps155137

Luppi T, Bas C, Méndez-Casariego A, Albano M, Lancia J, Kittlein M, Rosenthal A, Farías N, Spivak E, Iribarne O. 2013. The influence of habitat, season and tidal regime in the activity of the intertidal crab Neohelice (=Chasmagnathus) granulata. Helgol. Mar. Res. 67(1):1-15. https://doi.org/10.1007/s10152-012-0300-9

McLachlan A, Brown AC. 2006. The Ecology of Sandy Shores. Elsevier, USA, 392 pp. https://doi.org/10.1016/b978-0-12-372569-1.x5000-9

Melo WD. 2004. Orígenes morfológicos. In: Píccolo MC, Hoffmeyer MS (eds.), Ecosistema del Estuario de Bahía Blanca. Instituto Argentino de Oceanografía, Bahía Blanca, Argentina, pp. 21-27.

Merentiel-Ferreyra MN. 2014. Biología poblacional del cangrejo estuarino Neohelice granulata (Dana, 1851) (Crustacea: Brachyura: Varunidae) en los humedales del Santa Lucía, Montevideo. MSc thesis, Universidad de la República, Uruguay, $62 \mathrm{pp}$.

Milner RNC, Booksmythe I, Jennions MD, Backwell PRY. 2010. The battle of the sexes? Territory acquisition and defence in male and female fiddler crabs. Anim. Behav. 79(3): 735-738. https://doi.org/10.1016/j.anbehav.2009.12.030

Montemayor DI, Addino M, Fanjul E, Escapa M, Alvarez MF, Botto F, Iribarne OO. 2011. Effect of dominant Spartina species on salt marsh detritus production in SW Atlantic estuaries. J. Sea Res. 66(2): 104-110. https://doi.org/10.1016/j.seares.2011.05.003
Morrisey DJ, DeWitt TH, Roper DS, Williamson RB. 1999. Variation in the depth and morphology of burrows of the mud crab Helice crassa among different types of intertidal sediment in New Zealand. Mar. Ecol. Prog. Ser. 182: 231242. https://doi.org/10.3354/meps182231

MurrayJMH, Meadows A, Meadows PS.2002. Biogeomorphological implications of microscale interactions between sediment geotechnics and marine benthos: a review. Geomorphology 47(1): $15-30$. https://doi.org/10.1016/s0169-555x(02)00138-1

Negrín VL. 2011. El rol de las marismas del estuario de Bahía Blanca en el ciclo bio-geoquímico de nutrientes inorgánicos y de materia orgánica. Dissertation, Universidad Nacional del Sur, Argentina, 166 pp.

Piccolo MC, Perillo GME, Melo WD. 2008. The Bahía Blanca estuary: an integrated overview of its geomorphology and dynamics. In: Neves R, Baretta JW, Mateus M (eds.), Perspectives on Integrated Coastal Zone Management in South America. IST Press, Lisbon, pp. 219-230.

Qureshi NA, Saher NU. 2012. Burrow morphology of three species of fiddler crab (Uca) along the coast of Pakistan. Belg. J. Zool. 142(2): 114-126.

Sal-Moyano MP, Gavio MA, Luppi TA. 2012. Mating system of the burrowing crab Neohelice granulata (Brachyura: Varunidae) in two contrasting environments: effect of burrow architecture. Mar. Biol. 159(7): 1403-1416. https://doi.org/10.1007/s00227-012-1917-6

Spivak ED. 2010. The crab Neohelice (=Chasmagnathus) granulata: an emergent animal model from emergent countries. Helgoland Mar. Res. 64(3): 149-154. https://doi.org/10.1007/s10152-010-0198-z

Underwood AJ. 1997. Experiments in ecology: Their logical design and interpretation using analysis of variance. Cambridge Univ. Press, USA, 500 pp.

Zar JH. 1999. Biostatistical analysis. 4th ed. Prentice-Hall, Upper Saddle River (NJ).

Received November 2017, accepted April 2018. 